\title{
Disruption of the MDM2-p53 interaction strongly potentiates p53-dependent apoptosis in cisplatin-resistant human testicular carcinoma cells via the Fas/FasL pathway
}

\author{
R Koster ${ }^{1}$, H Timmer-Bosscha ${ }^{1}$, R Bischoff ${ }^{2}$, JA Gietema ${ }^{1}$ and S de Jong ${ }^{\star, 1}$
}

Wild-type p53 has a major role in the response and execution of apoptosis after chemotherapy in many cancers. Although high levels of wild-type p53 and hardly any TP53 mutations are found in testicular cancer (TC), chemotherapy resistance is still observed in a significant subgroup of TC patients. In the present study, we demonstrate that p53 resides in a complex with MDM2 at higher cisplatin concentrations in cisplatin-resistant human TC cells compared with cisplatin-sensitive TC cells. Inhibition of the MDM2-p53 interaction using either Nutlin-3 or MDM2 RNA interference resulted in hyperactivation of the p53 pathway and a strong induction of apoptosis in cisplatin-sensitive and -resistant TC cells. Suppression of wild-type p53 induced resistance to Nutlin-3 in TC cells, demonstrating the key role of p53 for Nutlin-3 sensitivity. More specifically, our results indicate that p53-dependent induction of Fas membrane expression ( threefold) and enhanced Fas/FasL interactions at the cell surface are important mechanisms of Nutlin-3-induced apoptosis in TC cells. Importantly, an analogous Fas-dependent mechanism of apoptosis upon Nutlin-3 treatment is executed in wild-type p53 expressing Hodgkin lymphoma and acute myeloid leukaemia cell lines. Finally, we demonstrate that Nutlin-3 strongly augmented cisplatin-induced apoptosis and cell kill via the Fas death receptor pathway. This effect is most pronounced in cisplatin-resistant TC cells.

Cell Death and Disease (2011) 2, e148; doi:10.1038/cddis.2011.33; published online 21 April 2011

Subject Category: Cancer

Most testicular cancer (TC) patients respond well to cisplatinbased chemotherapy; however, there is still a subset of these young patients that will die because of chemo-resistant or chemo-refractory disease. ${ }^{1}$ Similar to its effects in patients, cisplatin proved to be an extremely cytotoxic drug, inducing massive apoptosis in human TC cell lines. ${ }^{2-5}$ An important role of p53 in the response to chemotherapeutic drugs and the execution of apoptosis has been described. ${ }^{6}$ The p53 is a tumour suppressor protein with a dual role in stress response by transactivation of genes that induce apoptosis, such as FAS (TNFRSF6), as well as genes that induce cell-cycle arrest, such as cyclin-dependent kinase inhibitor $1 \mathrm{~A}$ gene (CDKN1A), encoding p21 cip1/waf1, allowing time for DNA repair. Function of p53 is regulated by several mechanisms, acting not only at the transcriptional and translational level, but also on stability, post-translational modification, and subcellular localisation of $\mathrm{p} 53 .^{7}$

Tumour protein p53 (TP53) is the most frequently mutated gene in human cancers. ${ }^{7,8}$ Surprisingly, in human TCs almost no TP53 mutations are found and wild-type p53 is expressed at high levels in the majority of TCs. ${ }^{9}$ Despite the increasing knowledge about p53 as a transactivator and cellular gatekeeper for cell growth and division, the effects of wild-type p53 (and mutated p53) on drug sensitivity of human tumours including TC are still not clear. We have previously shown that the response to cisplatin-induced DNA damage in TC cell lines is related to an induction of p53 expression and activation of the Fas death receptor pathway. ${ }^{2,9}$ Several other studies have reported the effect of wild-type p53 expression on chemo-sensitivity of human TC cell lines with contrasting and sometimes conflicting results. ${ }^{3,10-15}$

Tumours that retain wild-type p53 are supposed to have other defects in the p53 pathway, such as the presence of microRNA (miR)-371-373, miR-106b-seed-family members or cytoplasmic p21, the lack of phosphatase and tensin homologue (PTEN) expression or the increased mouse double minute 2 (MDM2) expression. ${ }^{16-19}$ MDM2, as transcriptional target of $\mathrm{p53}$, is the main negative feedback

\footnotetext{
${ }^{1}$ Department of Medical Oncology, University Medical Center Groningen, University of Groningen, Groningen, The Netherlands and ${ }^{2}$ Department of Analytical Biochemistry, University of Groningen, Groningen, The Netherlands

${ }^{*}$ Corresponding author: S de Jong, Department of Medical Oncology, University Medical Center Groningen, University of Groningen, Hanzeplein 1, 9713 GZ Groningen, The Netherlands. Tel + 31-(0)50-3612964; Fax + 31-(0)50-3614862; E-mail: s.de.jong@int.umcg.nl

Keywords: testicular cancer; wild-type p53; MDM2; Nutlin-3; Fas; cisplatin

Abbreviations: TC, testicular cancer; IP, immunoprecipitation; AML, acute myeloid leukaemia; miR, microRNA; RNAPII, RNA polymerase II, siRNA, small-interfering RNA; PBS, phosphate buffer saline; PE, phycoerythrin; MDM2, mouse double minute 2; TP53, tumour protein p53; FasL, Fas ligand; PTEN, phosphatase and tensin homologue; CDKN1A, cyclin-dependent kinase inhibitor 1A; RITA, reactivation of p53 and induction of tumours cell apoptosis; PARP, poly-(ADP-ribose) polymerase; $\angle R D D$, leucine-rich repeats and death domain containing; PHLDA, pleckstrin homology-like domain, family A, member 3; CDK2, cyclin-dependent kinase 2; ASPP1/2, apoptosis-stimulating of p53 protein 1/2; JMY, junction mediating and regulatory protein, p53 cofactor; HAUSP, herpes virus-associated ubiquitin-specific protease; NF-Y, nuclear transcription factor $Y$

Received 27.12.10; revised 09.3.11; accepted 14.3.11; Edited by A Stephanou
} 
regulator of $\mathrm{p53}$. By binding to the transactivation domain of p53, MDM2 is able to regulate p53 activity and stability via several mechanisms such as promoting p53 degradation through ubiquitination, stimulating p53 nuclear export, and inhibiting acetylation of $\mathrm{p} 53 .^{7}$

Interfering in the MDM2-p53 interaction, with small molecules like RITA and Nutlin-3, provides an attractive strategy for (re)activating wild-type p53 in a non-genotoxic way. This (re)activation leads to cell-cycle arrest and or apoptosis in tumour cells with wild-type p53. ${ }^{20-23}$ Restoration of p53 function by Nutlin-3 may thus have profound therapeutic effect on tumours that have retained wild-type p53, particularly if MDM2 activity is disproportionally increased. ${ }^{23}$ Recently, Nutlin-3-induced apoptosis was investigated in a small panel of TC cell lines, and only additive effects were seen in combination with cisplatin. However, no mechanistic insights in Nutlin-3-induced apoptosis were offered. ${ }^{24,25}$ In this study, we explore the potential of disrupting the MDM2-p53 interaction as a mean to activate p53 in TC. The role of p53 and MDM2 in cisplatin-induced apoptosis has been investigated using cisplatin-sensitive and -resistant human TC models. Finally, the importance of the Fas death receptor pathway in Nutlin-3 induced apoptosis has been studied.

\section{Results}

P53 and MDM2 cellular localisation and cisplatin response in TC Cells. In the present study, we have used a panel of cisplatin-sensitive and -resistant wild-type p53 expressing TC cell lines to compare cisplatin responses (Table 1) with the cellular localisation of p53 and MDM2, and MDM2-p53 complex formation (Figures 1a-c, Supplementary Figure 1). With immunofluorescence, we found that p53 is predominantly localised to the cytoplasm, while MDM2 was mainly present in the nucleus in all four cell lines (Figure 1a and Supplementary Figure 1). After exposure of cells to $8 \mu \mathrm{M}$ cisplatin, p53 became more nuclear localised, while MDM2 was observed in both the nucleus and cytoplasm of cisplatinsensitive Tera (Figure 1a) and 833KE (data not shown) cells. In contrast, in the intrinsically cisplatin-resistant TC cell lines, Scha, and 2102EP, and in Tera-CP, an acquired cisplatinresistant subline of Tera, p53 maintained localised in the cytoplasm, while MDM2 retained its nuclear localisation upon treatment with $8 \mu \mathrm{M}$ cisplatin (Supplementary Figure 1). Only at higher cisplatin concentrations, p53 became more nuclear localised, while MDM2 expression was then observed in both the nucleus and the cytoplasm (results not shown). Immunoprecipitation (IP) has been used to determine whether the observed shift in cellular localisation of p53 and MDM2 after cisplatin treatment affects MDM2-p53 complex formation (Figures $1 b$ and $c$ ). Note that we have used lower cisplatin concentrations for the cisplatin-sensitive Tera cell line compared with the cisplatin-resistant cell lines. Despite the strong induction of both p53 and MDM2 with increasing concentrations of cisplatin in Tera cells (lysates Figure $1 \mathrm{~b})$, IP experiments indicated a relative decrease in p53 being in complex with MDM2 in favour of unbound p53 upon increasing cisplatin concentrations (Figures $1 b$ and $c$ ). In contrast, sustained MDM2-p53 complex formation was still detected in cisplatin-resistant TC cells at relatively high cisplatin concentrations (up to $8 \mu \mathrm{M}$ ). Eventually, MDM2-p53 complexes in these cells were (partially) lost at cisplatin concentrations above $16 \mu \mathrm{M}$ (Figures $1 \mathrm{~b}$ and $\mathrm{c}$ ). Taken together, our results suggest that high sensitivity for cisplatin cytotoxicity and cisplatin-induced apoptosis is related to a reduction in MDM2-p53 complex formation and a change in p53 cellular localisation.

Apoptosis induction after disruption of the MDM2-p53 interaction depends on wild-type p53. To investigate the importance of the MDM2-p53 complex formation in preventing apoptosis in TC, we have used the small molecule inhibitors 'reactivation of p53 and induction of tumours cell apoptosis' (RITA) and Nutlin-3 that are supposed to disrupt the MDM2-p53 interaction. RITA induced massive apoptosis at nanomolar concentrations in the absence of transcriptional activation of any of the p53 targets tested (p53, MDM2, p21, and Fas). Furthermore, downregulation of $p 53$ with small-interfering RNA (siRNA) did not interfere with the apoptosis induction by RITA (Supplementary Figure 2a). Moreover, treatment with RITA induced caspase-dependent apoptosis in NCCIT, the TC cell line expressing mutant p53 (Supplementary Figure 2a), indicating that at least in TC cells RITA induces apoptosis independent of wild-type p53.

Therefore, further research was focused on the effects of the small molecule inhibitor Nutlin-3 in wild-type p53-expressing TC cells. Treatment of cisplatin-sensitive Tera and 833KE cells and cisplatin-resistant Tera-CP, Scha, and

Table $1 I^{\prime} C_{50}$ values and p53 status of the cell lines used in this study

\begin{tabular}{|c|c|c|c|c|}
\hline Cell line & $\mathrm{IC}_{50}$ cisplatin $(\mu \mathrm{M})^{\mathrm{a}}$ & $\mathrm{IC}_{50}$ combination $(\mu \mathrm{M})^{\mathrm{b}}$ & Enhancement ratio ${ }^{c}$ & P53 status ${ }^{d}$ \\
\hline $\begin{array}{l}\text { Tera } \\
\text { Tera-CP } \\
833 \mathrm{KE} \\
\text { Scha } \\
2102 \mathrm{EP} \\
\text { NCCIT }\end{array}$ & $\begin{array}{l}0.69 \pm 0.11 \\
2.14 \pm 0.17 \\
1.04 \pm 0.10 \\
2.91 \pm 0.84 \\
4.05 \pm 0.66 \\
2.15 \pm 0.34\end{array}$ & $\begin{array}{l}0.07 \pm 0.01 \\
0.11 \pm 0.02 \\
0.13 \pm 0.03 \\
0.28 \pm 0.08 \\
0.39 \pm 0.21 \\
2.25 \pm 0.59\end{array}$ & $\begin{array}{r}9.9 \\
19.5 \\
8.0 \\
10.4 \\
10.4 \\
1.0\end{array}$ & $\begin{array}{l}\mathrm{wt} / \mathrm{wt} \\
\mathrm{wt} / \mathrm{wt} \\
\mathrm{wt} / \mathrm{wt} \\
\mathrm{wt} / \mathrm{wt} \\
\mathrm{wt} / \mathrm{wt} \\
\mathrm{mt} / \mathrm{-}\end{array}$ \\
\hline
\end{tabular}

${ }^{\mathrm{a}} \mathrm{The}_{\mathrm{IC}}$ (drug concentration reducing cell survival by $50 \%$ ) for cisplatin was calculated from the graphs in Figure 6 a and Supplementary Figures $4 \mathrm{a}$ and b. The mean $\mathrm{IC}_{50} \pm$ S.D. was determined in three experiments, each performed in quadruplicate. ${ }^{\mathrm{b}} \mathrm{Th} \mathrm{IC}_{50}$ (drug concentration reducing cell survival by $50 \%$ ) for cisplatin in combination with $1 \mu \mathrm{M}$ Nutlin-3 was calculated from the graphs in Figure $6 \mathrm{a}$ and Supplementary Figures $4 \mathrm{a}$ and $\mathrm{b}$. The mean IC $\mathrm{F}_{50} \pm \mathrm{S}$.D. was determined in three experiments, each performed in quadruplicate. ${ }^{\mathrm{C}}$ Nutlin-3 enhancement ratios were calculated by dividing the $\mathrm{IC}_{50}$ for cisplatin alone by the IC $\mathrm{C}_{50}$ for the combination with cisplatin and $1 \mu \mathrm{M}$ Nutlin-3. ${ }^{\mathrm{d}}$ The p53 status of the cells was previously described ${ }^{3,30}$ 
a

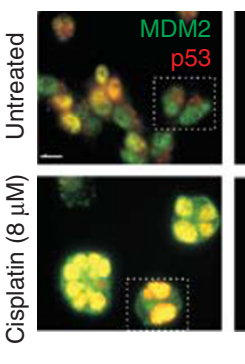

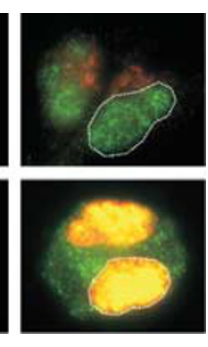

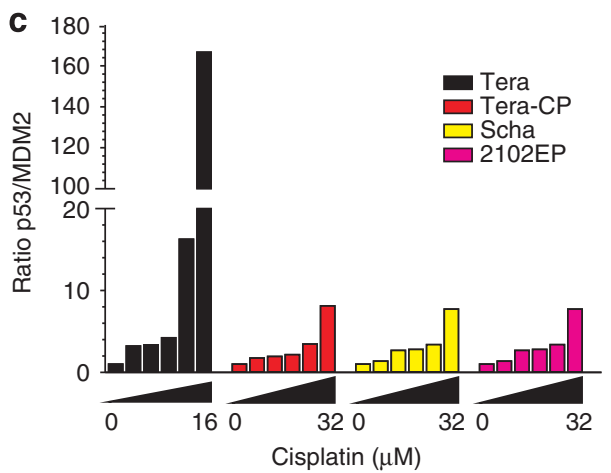

b
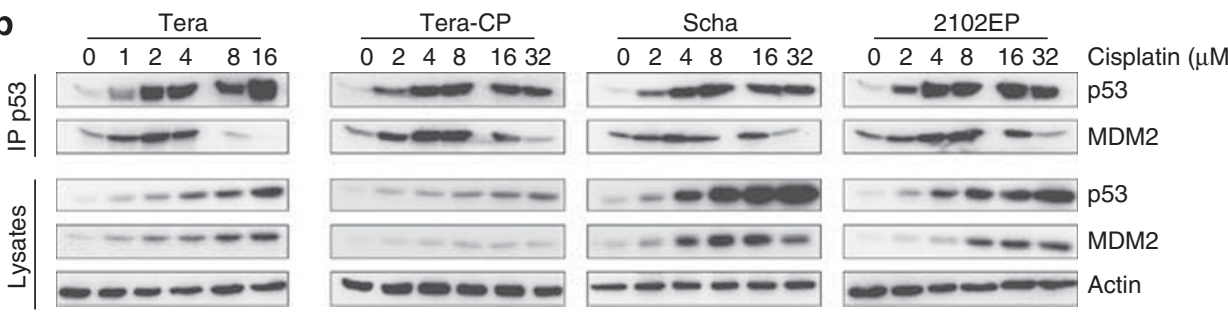

Figure 1 Earlier onset of cisplatin-induced loss of p53-MDM2 complex formation in cisplatin-sensitive TC cells. (a) Immunofluoresence showing that p53 becomes more nuclear localised, whereas MDM2 becomes nuclear and cytoplasmic localised after cisplatin treatment in the cisplatin-sensitive TC cell line Tera, representative example of three independent experiments. Selected area of the original image, as indicated, $\times 4$ digitally magnified. Scale bar: $30 \mu \mathrm{M}$. (b and $\mathbf{c})$ Note that we have used lower cisplatin concentrations for Tera compared with the other TC cell lines. TC cells were harvested $12 \mathrm{~h}$ after indicated cisplatin treatment. (b) Cell lysates were subjected to p53 IP. Immunoblotting was performed using anti-p53 and anti-MDM2 antibodies. In the cisplatin-resistant TC cell lines Tera-CP, 2102EP and Scha, p53 is maintained in a complex with MDM2 after cisplatin treatment, while the cisplatin-sensitive Tera cells show a loss of p53-MDM2 complex formation at low cisplatin concentrations. (c) Relative levels of p53 and MDM2 were calculated with imageJ 1.41 (National Institutes of Health, http://rsbweb.nih.gov/ij/index.html), normalised and divided p53/MDM2

a

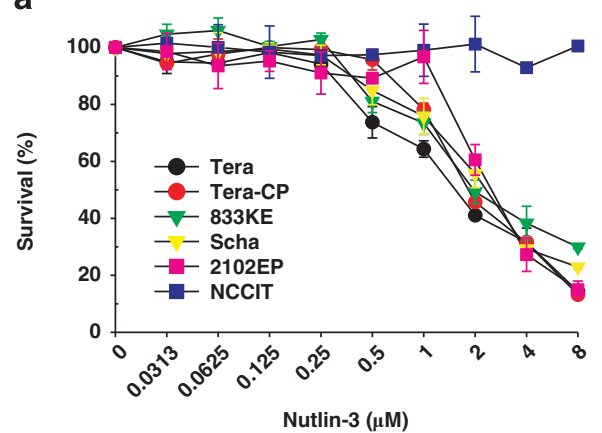

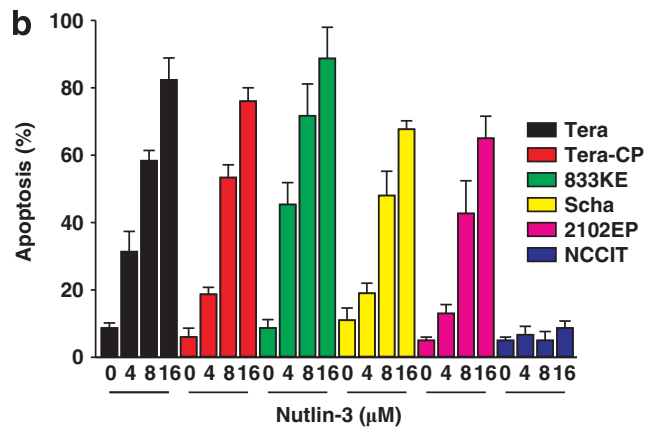

C
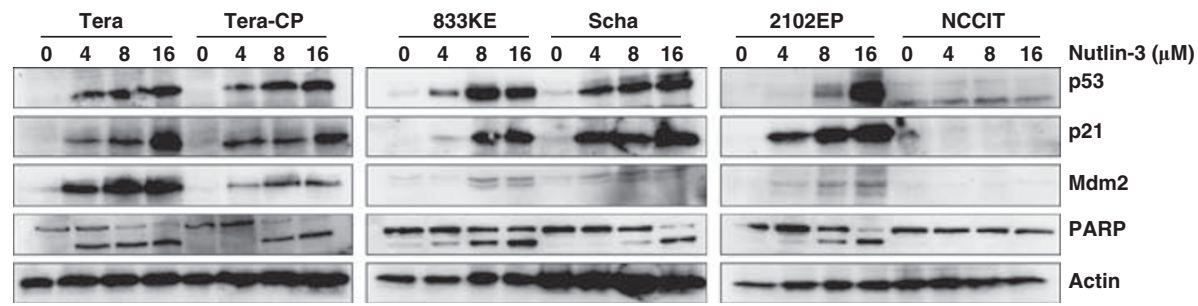

Figure 2 Nutlin-3 induces apoptosis in TC cells. (a) Survival of TC cells after $96 \mathrm{~h}$ of continuous Nutlin-3 treatment as indicated; values are the mean \pm S.D. of three independent experiments. (b) Apoptosis induction was analysed after Nutlin-3 treatment for $24 \mathrm{~h}$, by fluorescence microscopy on acridine orange-stained TC cells; values are the mean \pm S.D. of three experiments. (c) Immunoblot analysis showing upregulation of p53, MDM2 and p21 and enhanced cleavage of PARP and caspase-8 in wild-type p53 TC cells $24 \mathrm{~h}$ after Nutlin-3 treatment. The data presented are representative of three independent experiments

2102EP cells, all expressing wild-type p53, with Nutlin-3 resulted in a dose-dependent reduction in cell survival. No effect of Nutlin-3 on the survival of mutant p53-expressing NCCIT cells was observed (Figure 2a). The reduced survival after Nutlin-3 treatment is caused by a dose-dependent induction apoptosis (Figure $2 \mathrm{~b}$ ) that is caspase-dependent as reflected in the increased poly-(ADP-ribose) polymerase (PARP) cleavage (Figure 2c). Immunoblotting demonstrated 

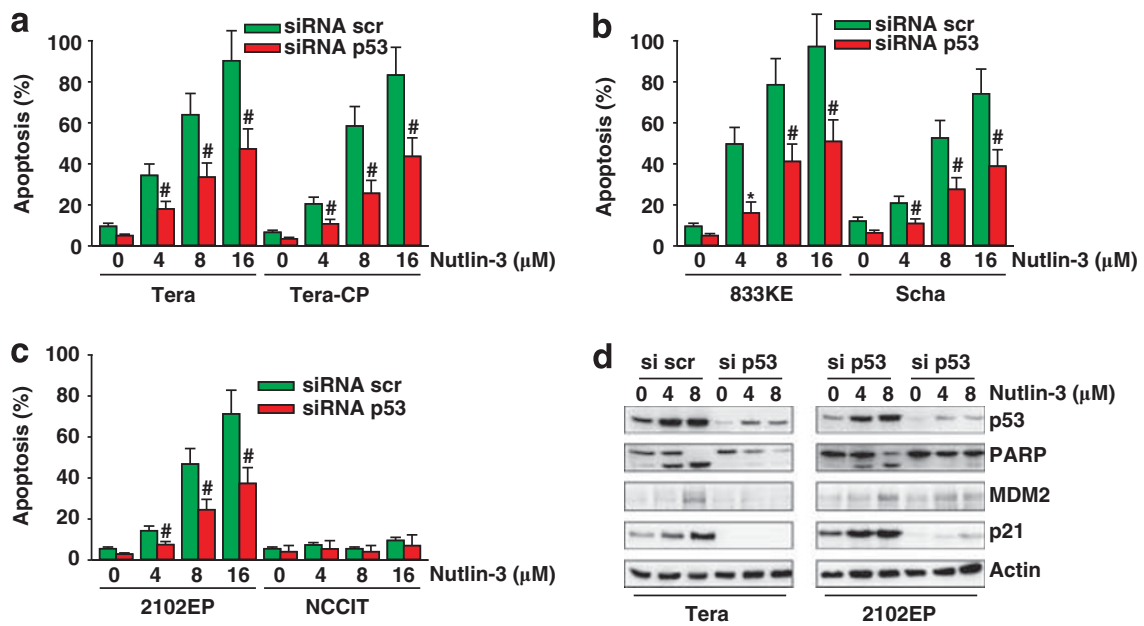

Figure 3 Wild-type p53 is necessary for the Nutlin-3 response. (a-c) Downregulation of p53 reduces the apoptotic response after Nutlin-3 treatment in wild-type p53expressing TC cells. Cells were treated with scrambled siRNA (siRNA scr) or with p53 siRNA (siRNA p53) for $24 \mathrm{~h}$. Values are the mean \pm S.D. of three experiments; ${ }^{\#} P<0.05 ;{ }^{*} P<0.01 ;{ }^{* *} P<0.005$. (d) Successful downregulation of $p 53$ using siRNA, decreases the expression of MDM2 and p21, and decreases PARP cleavage in Nutlin-3treated TC cells compared with control; a representative example of three independent experiments is shown

specific upregulation of $\mathrm{p} 53$ after treatment of the various wildtype p53-expressing TC cell lines with Nutlin-3. A dosedependant increase in the expression levels of the p53 transcriptional targets MDM2 and p21 was observed as well (Figure 2c). Furthermore, Nutlin-3 treatment led to a more pronounced nuclear localisation of p53 in all wild-type p53expressing TC cells (Supplementary Figure $2 b$ ). The mutant p53 NCCIT cell line, however, remained unaffected after treatment with Nutlin-3 (Figures $2 \mathrm{~b}$ and $\mathrm{c}$ ), suggesting a wildtype p53-dependent effect of Nutlin-3.

Next, we proved that the observed transcriptional activity and apoptosis induction after Nutlin-3 treatment are p53dependent effects, because suppression of p53 with p53 siRNA was accompanied by a reduced apoptotic response to Nutlin-3 treatment in all wild-type p53-expressing cells (Figures $3 \mathrm{a}-\mathrm{c}$ and Supplementary Figure $3 \mathrm{a}$ ). In addition, p53 suppression prevented the upregulation of MDM2 and p21 levels after Nutlin-3 treatment (Figure 3d). Transfection with the scrambled siRNA had no effect on p53 levels or Nutlin-3induced apoptosis, further demonstrating the wild-type p53-dependent effect of Nutlin-3 in TC cells.

Increased Fas death receptor expression in TC cells after Nutlin-3 treatment. Induction of the Fas death receptor membrane expression after drug treatment has been observed in several cell lines, which can occur in a p53dependent manner. ${ }^{2,5,26-30}$ In line with our previous results, ${ }^{2}$ we detected elevated Fas membrane expression in Tera cells after cisplatin treatment, whereas less induction was observed in Tera-CP cells and only a minor induction in 2102EP and Scha cells (Figures $4 a$ and b). Interestingly, Nutlin-3 treatment led to a robust upregulation of Fas membrane expression to levels significantly higher than found after solvent or cisplatin treatment in the wild-type p53-expressing TC cell lines (Figures 4a and b). Additionally, p53 suppression in wild-type p53 TC cells prevented the induction of Fas membrane expression by Nutlin-3 treatment
(Figure 4c). Induction of Fas membrane expression was not detected in the mutant p53-expressing NCCIT cells after cisplatin or Nutlin-3 treatment (Supplementary Figure $3 \mathrm{~b}$ ). These results prove that the observed upregulation of Fas membrane expression after Nutlin-3 treatment in TC cells is wild-type p53 dependent as well.

Fas-dependent apoptosis after Nutlin-3 treatment. We previously reported that cisplatin-induced apoptosis is depending on activation of the Fas/ Fas Ligand (FasL) system in cisplatin-sensitive TC cells. ${ }^{2}$ Activation of the Fas/ FasL system was largely impaired in the cisplatin-resistant Tera-CP and Scha cells. ${ }^{2}$ To further investigate the importance of Fas upregulation for Nutlin-3-induced apoptosis, we inhibited the Fas-FasL interaction by either blocking FasL with FasL-specific NOK-1 antibody or downregulation of FasL with FasL siRNA. Effective downregulation of FasL was confirmed by immunoblotting (Figure 4d and Supplementary Figure 3c). Blocking of FasL and suppression of FasL dramatically reduced the apoptotic response after high doses Nutlin-3 in the wild-type p53expressing TC cell lines (Figure 4e), as visualised by a significant decrease in active, cleaved caspase-8 and PARP cleavage (Figure $4 \mathrm{~d}$ and Supplementary Figure 3c). Interfering in the Fas/FasL system, however, had no effect on the induction of p53 by Nutlin-3 (Figure 4d and Supplementary Figure $3 \mathrm{c}$ ). Our results demonstrate that the massive apoptosis induction in TC cells after Nutlin-3 treatment is to a large extent dependent on the activation of the Fas death receptor pathway.

We have demonstrated that high levels of cytoplasmic localised p21 protected Scha and 2102EP cells against cisplatin-induced apoptosis, probably by interfering with Fas-death receptor signalling. ${ }^{19,30}$ In this study, we show that treatment with a relatively low dose of Nutlin-3 $(4 \mu \mathrm{M})$ led to higher levels of cytoplasmic localised p21 that was most pronounced in 2102EP and Scha cells (Figure 2c and 


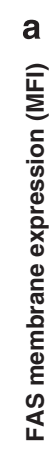

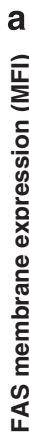

d

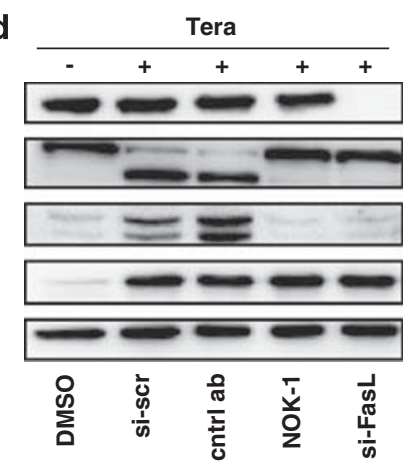

b

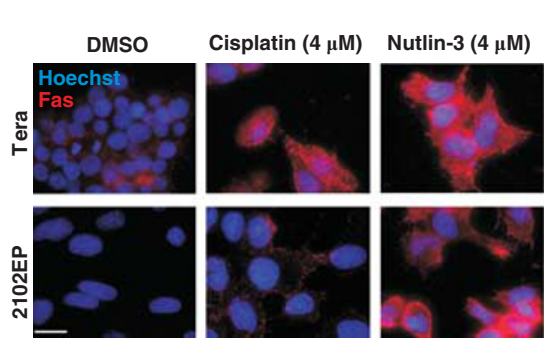

C
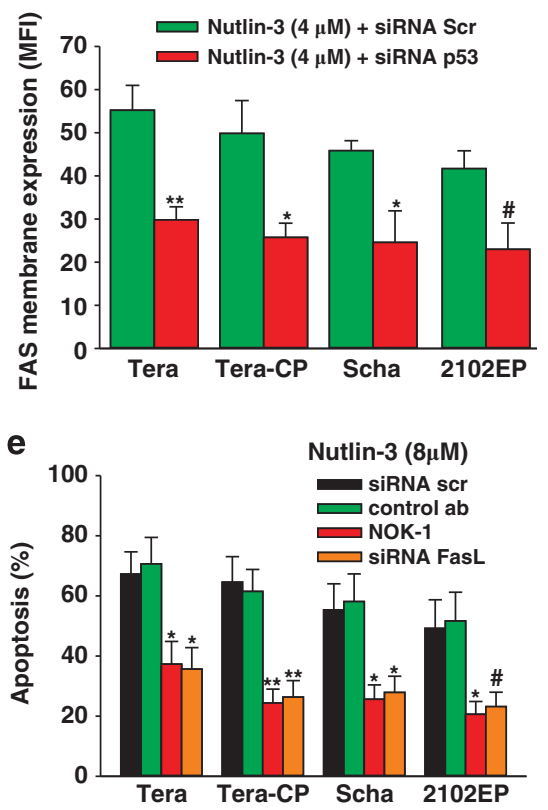

Figure 4 Nutlin-3 treatment induces Fas-dependent apoptosis in TC cells. (a) Following the indicated treatment, TC cells were harvested and Fas membrane expression was determined by flow cytometry. Values are depicted as MFI. Values are the mean \pm S.D. of three experiments. (b) TC cells were treated as indicated and Fas-membrane expression has been determined by immunofluorescence, representative example of three independent experiments. Scale bar: $30 \mu \mathrm{M}$. (c) Downregulation of p53 reduces Fas membrane expression after Nutlin-3 treatment in wild-type p53-expressing TC cells, compared with control; values are the mean \pm S.D. of three experiments; ${ }^{\#} P<0.05$; ${ }^{\star} P<0.01 ;{ }^{\star \star} P<0.005$. (d) Fas acts pro-apoptotic after $24 \mathrm{~h}$ Nutlin-3 treatment in TC cells. After successful downregulation of FasL or blocking of FasL with NOK-1, a decrease in PARP cleavage and active caspase-8 is observed in Tera and 2102EP; a representative example of three independent experiments is shown. (e) Decreased apoptotic response after blocking or suppression of FasL, in TC cells treated with Nutlin-3; values are the mean \pm S.D. of three experiments

Supplementary Figures $3 d$ and e). Using the same Nutlin-3 concentration, a concomitantly lower apoptotic response was observed in the cisplatin-resistant Scha and 2102EP cells compared with the cisplatin-sensitive TC cells (Figure 2b). Treatment with high-dose Nutlin-3 further increased the fold of induction of p21 in the wild-type p53-expressing TC cells, but p21 seems to no longer be able to inhibit apoptosis induction. Therefore, the importance of cytoplasmic p21 in inhibiting Nutlin-3-induced apoptosis has been further defined using an siRNA approach. Downregulation of p21 (Supplementary Figure 3e) led to an increase in Nutlin-3-induced apoptosis (at $4 \mu \mathrm{M}$ ) in Scha and 2102EP cells, as demonstrated by an increase in PARP cleavage (Supplementary Figure 3e). Thus, p21 can have a role in inhibiting Nutlin-3-induced apoptosis.

Next, we addressed the question, if the Fas death receptor pathway also has an important role in Nutlin-3-induced apoptosis in other non-testicular tumour cell types. To this end, we analysed Hodgkin lymphoma and acute myeloid leukaemia (AML) cell lines, which are also known to be sensitive to Nutlin-3. ${ }^{21,22}$ Blocking of FasL with NOK-1 considerably reduces the apoptotic response and PARP cleavage after Nutlin-3 treatment in the wild-type p53expressing Hodgkin lymphoma cell lines KM-H2 and L540 (Figure 5a), as well as in the wild-type p53-expressing MOLM13 AML cells (Figure 5b). In addition, no effect of either Nutlin-3 treatment or blocking of the Fas/FasL interaction was
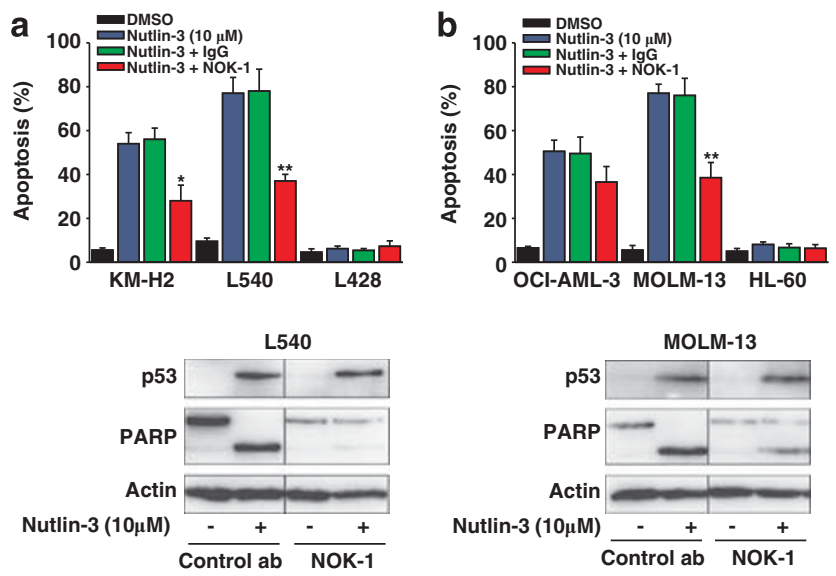

Figure 5 Nutlin-3 treatment induces Fas-dependent apoptosis in haematological neoplasm. (a and $\mathbf{b})$ Decreased apoptotic response and reduced PARP cleavage after blocking of FasL in wild-type p53-expressing Hodgkin lymphoma cells (a) and AML cells (b) after Nutlin-3 treatment. Values are the mean \pm S.D. of three experiments; ${ }^{\star} P<0.01 ;{ }^{*} P<0.005$

observed in OCl-AML3 or the mutant p53 expressing Hodgkin lymphoma and AML cell lines, L428 (Figure 5a) and HL-60 (Figure $5 b$ ), respectively. This shows that the Fas death receptor pathway also has an important role in Nutlin-3induced apoptosis in wild-type p53 expressing cell lines derived from other tumour types. 
a

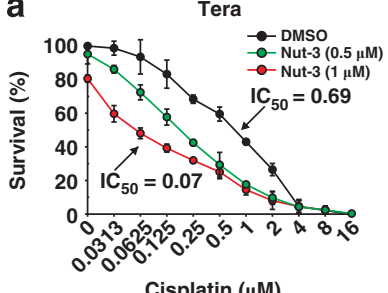

Cisplatin $(\mu \mathrm{M})$

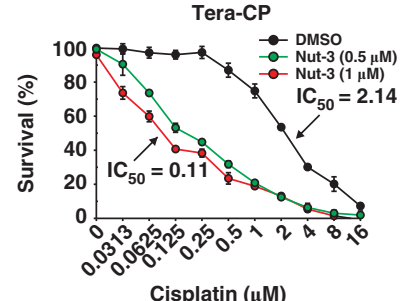

Cisplatin $(\mu \mathrm{M})$

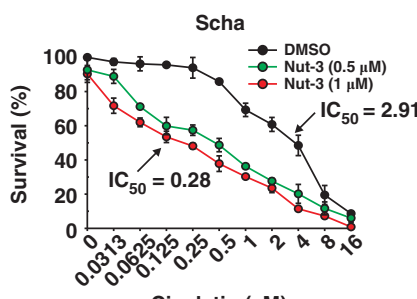

Cisplatin $(\mu \mathrm{M})$
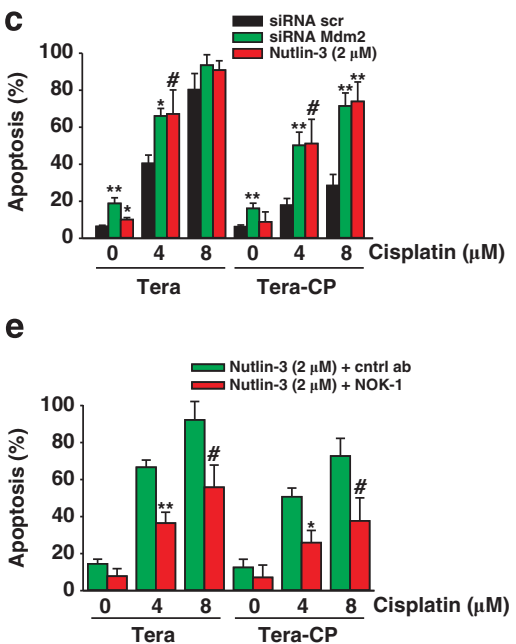

Figure 6 Synergistic effect of combining cisplatin with inhibition of the MDM2-p53 interaction. (a) Survival of TC cells after $96 \mathrm{~h}$ of continuous Nutlin-3 (Nut-3) treatment as indicated, in combination with increasing cisplatin concentration. $\mathrm{IC}_{50}$ values are depicted for cisplatin alone as well as the combination of cisplatin with $1 \mu \mathrm{M}$ Nutlin-3; values are the mean \pm S.D. (b) Increased levels of p53 and increased PARP cleavage after targeting the MDM2/p53 axis; a representative example of three independent experiments is shown. (c) Increased apoptosis after targeting the MDM2/p53 axis with either siRNA against MDM2 or Nutlin-3 (Nut-3) in combination with cisplatin; values are the mean \pm S.D. of three experiments; ${ }^{\#} P<0.05$; ${ }^{\star} P<0.01$; ${ }^{\star \star} P<0.005$. (d) Following indicated treatment TC cells were harvested and Fas membrane expression has been determined by flow cytometry. Values are depicted as MFI. Values are the mean \pm S.D. of three experiments (e) Decreased apoptotic response after blocking of FasL, with NOK-1, in TC cells treated with the combination of cisplatin and Nutlin-3 as indicated; values are the mean \pm S.D. of three experiments; ${ }^{\#} P<0.05 ;{ }^{*} P<0.01 ;{ }^{\star \star} P<0.005$

\section{Synergistic effect of Nutlin-3 and cisplatin is Fas death} receptor and p53-dependent. Finally, we tested the cytotoxicity of cisplatin in combination with minimally toxic concentrations of Nutlin-3 in TC cells. Treatment for $96 \mathrm{~h}$ with a combined drug treatment led to much stronger reductions in survival of all wild-type p53 TC cell lines tested, as compared with single drug treatment with cisplatin or Nutlin-3 (Figure 6a, Supplementary Figure 4a). The strongest decrease in survival, combining the two drugs, has been observed in the intrinsic and acquired cisplatin-resistant cell lines (Figure 6a, Supplementary Figure 4a and Table 1). As expected, the combination with Nutlin-3 has no potentiating effect on cisplatin-induced cytotoxicity in the mutant p53 cell line NCCIT (Supplementary Figure 4b).

The mechanism of sensitisation has been further investigated in the acquired cisplatin-resistant cell model (Figures $6 \mathrm{~b}$ and e) and intrinsic cisplatin-resistant cell lines (Supplementary Figure 5). Combined treatment with Nutlin-3 and cisplatin strongly enhanced p53 upregulation compared with the effect of either cisplatin or Nutlin-3 treatment alone on p53 levels. This was accompanied by a large induction of apoptosis as reflected in almost complete PARP cleavage with the combination and minimal PARP cleavage with either drug alone, which was most contrasting for Tera-CP (Figures $6 \mathrm{~b}$ and c) and Scha and 2102EP (Supplementary Figures 5a and b).
To investigate if the Fas death receptor pathway also has an important role in apoptosis-induction after combination treatment, Fas membrane expression levels were determined. Nutlin-3 in combination with cisplatin resulted in much higher Fas membrane expression levels compared with the levels found after treatment with Nutlin-3 or cisplatin alone in wildtype p53-expressing TC cell lines (Figure 6d and Supplementary Figure $5 \mathrm{c}$ ). No induction of Fas membrane expression was observed in the mutant p53-expressing NCCIT after the various treatments (Supplementary Figure $3 \mathrm{~b}$ ). Additionally, blocking of FasL with NOK-1 reduced the apoptotic response of wild-type p53-expressing TC cells to the combined treatment of Nutlin-3 and cisplatin (Figure 6e and Supplementary Figure $5 d$ ). Suppression of MDM2 with siRNA extremely sensitised TC cells to cisplatin-induced apoptosis, almost similar to our observations with Nutlin-3. Moreover, p53 expression and Fas membrane expression were strongly upregulated after treatment with MDM2 siRNA in combination with cisplatin, especially in the cisplatin-resistant TC cells. This demonstrates that the release of the negative feedback on p53 by MDM2 is the important event for enhancing cisplatin-induced apoptosis in TC cells (Figures $6 b-d$ and Supplementary Figures $5 \mathrm{a}-\mathrm{c}$ ).

Taken together, these results indicate that targeting MDM2 in combination with cisplatin treatment overcomes both 
intrinsic as well as acquired-resistance to cisplatin in wild-type p53-expressing TC cells, and is largely dependent on activation of the Fas death receptor pathway.

\section{Discussion}

In the present study, we demonstrate that wild-type p53 is sustained in complex with MDM2 in cisplatin-resistant TC cell lines following treatment with therapeutically relevant cisplatin concentrations. Our results indicate that interfering in the MDM2-p53 interaction through the small molecule compound Nutlin-3, sensitises wild-type p53-expressing TC cells for apoptosis. Combining Nutlin-3 with cisplatin, the most important therapeutic drug in the treatment of TC patients, results in hyper-activation of the p53 pathway, largely sensitises both intrinsic as well as acquired cisplatin-resistant TC cells to apoptosis via the Fas/FasL death receptor pathway, and strongly reduces cell survival. These results indicate that targeting the MDM2/p53 axis, in combination with standard cisplatin-based chemotherapeutic treatment, is an attractive therapeutic strategy to pursue for cisplatin-resistant/ refractory TC (Figure 7).

P53-interacting proteins, such as MDM2, are important regulators of wild-type p53 functionality. ${ }^{7,20}$ The lack of TP53 mutations in TC has led to the hypothesis that constitutively expressed p53 is functionally inactive. ${ }^{31}$ Surprisingly high levels of wild-type p53 have been frequently observed in TC. These levels correlate with expression levels of the p53 transcriptional target MDM2, suggesting that p53 is functional in TC. ${ }^{11,13,17}$ In this study, we show that treatment with the selective MDM2 antagonist Nutlin-3 causes a high induction of both p53 and MDM2, a massive induction of apoptosis, and a strong reduction in cell survival in cisplatin-sensitive as well as cisplatin-resistant TC cell lines. In addition, siRNA targeting MDM2 had similar effects as Nutlin-3 treatment, indicating an important role of MDM2 as a negative-feedback regulator of
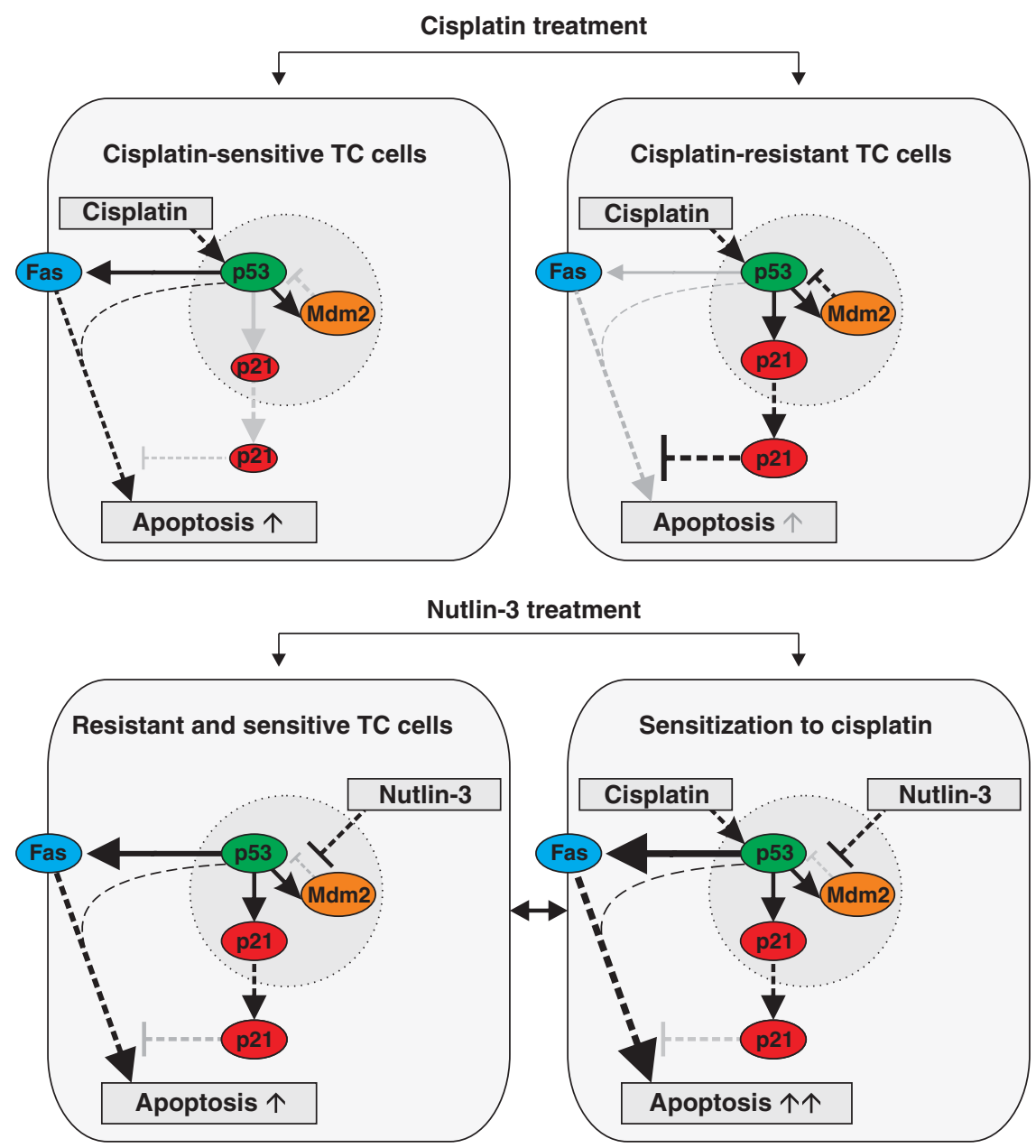

Figure 7 Simplified model showing the role of the MDM2/p53 axis in regulating the sensitivity to cisplatin and Nutlin-3 in wild-type p53-expressing TC cells. P53 is a transcriptional activator of p21, Fas and MDM2. Cisplatin sensitive TC cells have low levels of p21 and cisplatin-resistant TC cells, due to higher CDKN1A expression levels and lower levels of Oct4 and miR-106b family members, high levels of cytoplasmic p21, which is a key determinant of resistance to cisplatin-induced apoptosis. ${ }^{19}$ Cisplatininduced apoptosis in TC cells also involves activation of the Fas death receptor pathway via elevated Fas membrane expression. High cytoplasmic p21 levels inhibit Fas death receptor-mediated apoptosis in cisplatin-resistant TC cells. ${ }^{30}$ Moreover, cisplatin-induced DNA damage activates p53 and enhances release of p53 from MDM2-p53 complex, while sustained MDM2-p53 complex formation is found in cisplatin-resistant cells. Interfering in MDM2-p53 complex formation by Nutlin-3 treatment (or suppression of MDM2) substantially induces Fas expression, resulting in apoptosis of both cisplatin sensitive and resistant TC cells. Cisplatin in combination with Nutlin-3 further enhances Fas expression and sensitises cisplatin-sensitive and resistant TC cells to cisplatin-induced apoptosis. Dotted lines indicate interaction or signaling. Solid lines indicate p53dependent transcription. Grey dotted and solid lines specify reduced activity 
p53 activity, however we cannot exclude a role for Nutlin-3induced release of MDM2 in the observed apoptosis. These results suggest a tight regulation of MDM2 interacting with p53. Several in vitro and in vivo studies suggest an important role for p53 in the cisplatin-sensitivity of TC. ${ }^{10,11,15,31,32}$ In contrast, other studies have failed to support a role for p53 in TC responsiveness, ${ }^{13}$ although the involvement of the MDM2-p53 complex in the response to chemotherapy had not been thoroughly assessed. ${ }^{13,14}$ The present study indicates that $\mathrm{p} 53$ function is impeded by the interaction with MDM2 and the subsequent sequestration of p53 in the cytoplasm of cisplatin-resistant TC cells following cisplatin treatment. The strong sensitisation to cisplatin by low doses of Nutlin-3 further demonstrates the importance of MDM2 in controlling p53 following cisplatin-induced DNA damage.

Induction of FasL and upregulation of the Fas receptor in a p53-dependent manner has been observed in several tumour cell lines after treatment with chemotherapeutic drugs, such as cisplatin, and is related to apoptosis induction. ${ }^{2,5,26-30}$ Previously, we have reported that the Fas/FasL system is active and functional in cisplatin-sensitive but almost inactive in cisplatin-resistant TC cell lines. ${ }^{2}$ In the present study, we show that Nutlin-3 treatment strongly enhanced Fas membrane expression levels in TC cells. Fascinatingly, blocking of the Fas-FasL interaction in TC cells impairs apoptosis induction by Nutlin-3 as well as combined treatment with Nutlin-3 and cisplatin. However, involvement of other genes in this pathway cannot be excluded, because p53 has been shown to transcriptionally activate several other genes involved in apoptosis via the Fas/FasL system after cisplatin treatment. In the cisplatin-sensitive Tera cells, besides FAS, the Fas adaptor leucine-rich repeats and death domain containing $(L R D D)$ and a gene implicated in positive Fas regulation, pleckstrin homology-like domain, family $A$, member 3 (PHLDA3) were found to be regulated by p53 upon cisplatin treatment. ${ }^{15}$ Importantly, we have observed that the Fas/FasL system also has an important role in Nutlin-3induced apoptosis of wild-type p53-expressing AML and Hodgkin cell lines. The wild-type p53-expressing cell line $\mathrm{OCl}$ AML-3, however, did not show a significant reduction in Nutlin3-induced apoptosis after FasL blocking. This latter result can be explained by the observation that Nutlin-3-induced apoptosis was independent of transcriptional activation of p53 in these cells. ${ }^{21}$ Our results, thus, indicate a major role for the Fas/FasL system in the response to Nutlin-3 of wild-type p53-expressing TC, AML, and Hodgkin cell lines.

We have recently reported the important protective role of elevated p21 levels in cisplatin-resistant TC cells as compared with p21 levels in cisplatin-sensitive TC cells. ${ }^{19,30}$ In cisplatinresistant TC cells, p21 is cytoplasmic localised thus inhibiting cisplatin-induced cyclin-dependent kinase 2 and Fasmediated apoptosis. In addition, cisplatin- or irradiationinduced upregulation of p21 does not induce cell-cycle arrest in TC cells. ${ }^{19,30}$ The present results indicate that cytoplasmic localised p21 blocks Nutlin-3-induced apoptosis in cisplatinresistant TC cells that are also less sensitive to Nutlin-3 compared with cisplatin-sensitive TC cells. Xia et al. ${ }^{33}$ has not found a protective role of p21 against Nutlin-3-induced apoptosis in non-TC cell lines, though cellular localisation of p21 was not assessed. The p53-dependent induction of p21 in these non-TC cells by Nutlin-3 resulted in a cell-cycle arrest, ${ }^{33}$ strongly suggesting nuclear localisation of $\mathrm{p} 21$. In contrast, in TC cells Nutlin-3 treatment did not induce cell-cycle arrest, ${ }^{24,25}$ probably as a result of the predominantly cytoplasmic localisation of p53-dependent p21 expression by Nutlin-3.

Treatment with higher Nutlin-3 concentrations led to a strong induction of p53, a further increase in Fas membrane expression and higher levels of apoptosis in cisplatinsensitive and -resistant TC cells. Non-apoptotic genes, such as $C D K N 1 A$, constitutively harbour high levels of the poised RNA polymerase II (RNAPII) initiation complex at their core promoters, which are converted into elongated forms shortly after stress, but reinitiate very poorly. In contrast, proapoptotic genes, including FAS, have low levels of bound RNAPII but undergo damage-induced activation through multiple rounds of efficient reinitiation. ${ }^{34-36}$ Additionally, the co-factors apoptosis-stimulating of p53 protein 1/2 (ASPP1/2), junction mediating and regulatory protein, p53 cofactor (JMY), herpes virus-associated ubiquitin-specific protease (HAUSP), and nuclear transcription factor $Y$ (NF-Y) enhance p53 apoptotic activity by facilitating its binding to pro-apoptotic promoters. $^{7,35,36}$ For instance, NF-Y is known to be an essential positive regulator of $F A S$ transcription, whereas it represses $C D K N 1 A$ promoter activity. ${ }^{35,36}$ Interestingly, p53 occupancy of the p21 promoter in TC cells was similar to the levels observed in non-TC cells, whereas much less p21 expression was found in TC cells. ${ }^{25}$ In addition, p21 mRNA and protein levels remained relatively low after either Nutlin- $3^{25}$ or cisplatin treatment in TC cells as compared with other cancer cell types. ${ }^{2,19,25,30}$ Therefore, it is tempting to speculate that the Nutlin-3-induced release of the negative feedback on $\mathrm{p} 53$ by MDM2 further shifts the balance towards transcribing pro-apoptotic genes, for instance involved in the Fas death receptor pathway. This effect may be further enhanced by the combination of Nutlin-3 with cisplatin.

A previous study has reported that combining Nutlin-3 with other cytotoxic agents enhances the activity of these agents in wild-type p53-expressing leukaemia cells. ${ }^{21}$ Our results show that combining non-toxic concentrations of Nutlin-3 with cisplatin sensitises both intrinsic as well as acquired cisplatin-resistant TC cells to low concentrations of cisplatin. Of interest, MDM2 inhibitors in contrast to cisplatin might be considered non-genotoxic, as demonstrated with Nutlin-3 in mice. ${ }^{23}$ Nutlin-3 even showed protective activity in normal kidney cells against cisplatin-induced apoptosis. ${ }^{37}$ MDM2 inhibitors have entered phase I clinical trials in haematological malignancies and solid tumours. Based on the present results, clinical trials using MDM2 inhibitors such as Nutlin-3 in combination with cisplatin to treat cancer patients with wildtype $\mathrm{p53}$, for instance refractory TC patients, may be of great importance.

In conclusion, we demonstrate that the negative feedback regulator MDM2 has an important role in the sensitivity of wild-type p53-expressing TC cells to Nutlin-3 and cisplatin. Our findings show that disrupting the MDM2-p53 interaction, also in combination with cisplatin, enhances Fas death receptor-mediated apoptosis in TC cells. Targeting the MDM2/p53 axis in combination with standard cisplatin-based 
treatment is a therapeutic strategy warranted to pursue in cisplatin-resistant/refractory TCs.

\section{Materials and Methods}

Cell lines and reagents. A well-defined panel of cisplatin-sensitive and resistant EC cell lines 833KE, Tera, Tera-CP, Scha, and 2102EP (all expressing wild-type p53) and NCCIT (mutant p53) were used in this study. ${ }^{3,19,30,38,39}$ Tera Tera-CP, 2102EP, Scha, 833KE, and NCCIT were cultured and harvested as described previously. ${ }^{19}$ The cell lines OCI-AML-3, MOLM-13, HL-60, KM-H2, L540, and $L 428$ were maintained as described previously. ${ }^{21,22}$ Cisplatin was purchased from Bristol-Myers Co. (Weesp, the Netherlands), Nutlin-3 from Cayman Chemical (Huissen, the Netherlands). To block Fas/FasL interactions, cells were incubated with anti-FasL Ab NOK-1 (Becton Dickinson, Breda, the Netherlands) and azidefree IgG control (Becton Dickinson).

Drug sensitivity assay. Drug sensitivity testing was performed with the microculture tetrazolium assay as described previously. ${ }^{19}$

Immunofluorescence. Cells were seeded on $0.01 \%$ poly-L-lycine (Sigma, Amsterdam, The Netherlands) pre-coated coverslips. After $24 \mathrm{~h}$, indicated treatment cells were fixed with methanol/acetone $(1: 1)$ for $30 \mathrm{~min}$ at RT and then blocked with $1 \%$ bovine serum albumin and $1 \%$ normal goat serum in PBS for 30 min at RT. Followed by immunostaining with the corresponding antibodies and counterstained with Alexa-Fluor goat secondary antibodies (Molecular Probes, Invitrogen, Merelbeke, Belgium). Finally, cells were stained with Hoechst 33258 (Molecular Probes, Invitrogen) for $5 \mathrm{~min}$, washed with PBS, and coverslips were mounted on slides with Vectashield (Vector Laboratories, Amsterdam, the Netherlands). After staining cells were analysed using a Quantimet 600 S digital analysis system (Leica Microsystems, Rijswijk, The Netherlands).

IP. IP was performed with a mixture of agarose conjugated anti-p53 (D01 \& FL-393, Santa Cruz, CA, USA) as described previously. ${ }^{19}$

SDS-polyacrylamide gel electrophoresis and immunoblotting. After $24 \mathrm{~h}$, indicated treatment cells were harvested and lysates were examined by WB as described previously. ${ }^{19,30}$ Antibodies used are listed in Supplementary Methods.

Apoptosis. Cells were continuously incubated with cisplatin for $24 \mathrm{~h}$ at various concentrations. Acridine orange fluorescent staining of nuclei in unfixed cells was used to distinguish apoptotic from vital cells. ${ }^{19,30,40}$

RNA interference. The siRNA specific for human p53, MDM2, FasL, and negative control (scrambled) were purchased from Eurogentec (Maastricht, the Netherlands). TC cells were transfected in six-well plates with $5 \mu$ l of $20 \mu \mathrm{M}$ siRNA duplex or siRNA anti-sense using Oligofectamine reagent according to the manufacturer's instructions (Invitrogen, Merelbeke, Belgium). After $24 \mathrm{~h}$, cells were treated with cisplatin. $24 \mathrm{~h}$ after the treatment cells were harvested for protein isolation. Alternatively, in order to perform an apoptosis assay, at $24 \mathrm{~h}$ after transfection, cells were harvested and plated in 96-well plate. The day after, cells were treated with cisplatin. All sequences are listed in Supplementary Methods.

Fas-membrane expression. TC cells were treated as indicated and eventually stained with a phycoerythrin (PE)-conjugated Ab against Fas (DX2, Becton Dickinson) for $1 \mathrm{~h}$ at room temperature. Subsequently, cells were washed and analysed by flow cytometry (FACS-Calibur; Becton Dickinson). The mean fluorescence intensity (MFI) was determined by comparison of the fluorescence intensity of unlabelled cells.

Statistical analysis. Results of at least three experiments are expressed as mean \pm (standard deviation) S.D. Student's unpaired $t$-test was used to compare values of test and control samples. All tests were two-sided and differences were considered to indicate significance when $P<0.05$.

\section{Conflict of interest}

The authors declare no conflict of interest.
Acknowledgements. We wish to thank Theo Plantinga and Marieke Smit for technical support and Marcel van Vugt for critical reading of the manuscript.

1. Horwich A, Shipley J, Huddart R. Testicular germ-cell cancer. Lancet 2006; 367: 754-765.

2. Spierings DC, de Vries EG, Vellenga E, de Jong S. Loss of drug-induced activation of the CD95 apoptotic pathway in a cisplatin-resistant testicular germ cell tumor cell line. Cell Death Differ 2003; 10: 808-822.

3. Burger H, Nooter K, Boersma AW, van Wingerden KE, Looijenga LH, Jochemsen AG et al. Distinct p53-independent apoptotic cell death signalling pathways in testicular germ cell tumour cell lines. Int J Cancer 1999; 81: 620-628.

4. Walker MC, Parris CN, Masters JR. Differential sensitivities of human testicular and bladder tumor cell lines to chemotherapeutic drugs. J Natl Cancer Inst 1987; 79: 213-216.

5. di Pietro A, Vries EG, Gietema JA, Spierings DC, de Jong S. Testicular germ cell tumours: the paradigm of chemo-sensitive solid tumours. Int J Biochem Cell Biol 2005; 37: 2437-2456.

6. Johnstone RW, Ruefli AA, Lowe SW. Apoptosis: a link between cancer genetics and chemotherapy. Cell 2002; 108: 153-164.

7. Vousden KH, Prives C. Blinded by the light: the growing complexity of p53. Cell 2009; 137 : 413-431.

8. Hamroun D, Kato S, Ishioka C, Claustres M, Beroud C, Soussi T. The UMD TP53 database and website: update and revisions. Hum Mutat 2006; 27: 14-20.

9. Houldsworth J, Korkola JE, BosI GJ, Chaganti RS. Biology and genetics of adult male germ cell tumors. J Clin Oncol 2006; 24: 5512-5518.

10. Chresta CM, Arriola EL, Hickman JA. Apoptosis and cancer chemotherapy. Behring Inst Mitt 1996; 97: 232-240.

11. Houldsworth J, Xiao H, Murty VV, Chen W, Ray B, Reuter VE et al. Human male germ cell tumor resistance to cisplatin is linked to TP53 gene mutation. Oncogene 1998; 16: 2345-2349.

12. Arriola EL, Rodriguez-Lopez AM, Hickman JA, Chresta CM. Bcl-2 overexpression results in reciprocal downregulation of $\mathrm{Bcl}-\mathrm{X}(\mathrm{L})$ and sensitizes human testicular germ cell tumours to chemotherapy-induced apoptosis. Oncogene 1999; 18: 1457-1464.

13. Kersemaekers AM, Mayer F, Molier M, van Weeren PC, Oosterhuis JW, Bokemeyer C et al. Role of P53 and MDM2 in treatment response of human germ cell tumors. J Clin Oncol 2002; 20: 1551-1561.

14. Oliver RT, Shamash J, Berney DM. p53 and MDM2 in germ cell cancer treatment response. J Clin Oncol 2002; 20: 3928; author reply 3928-3929.

15. Kerley-Hamilton JS, Pike AM, Li N, DiRenzo J, Spinella MJ. A p53-dominant transcriptional response to cisplatin in testicular germ cell tumor-derived human embryonal carcinoma. Oncogene 2005; 24: 6090-6100.

16. Di Vizio D, Cito L, Boccia A, Chieffi $P$, Insabato $L$, Pettinato $G$ et al. Loss of the tumor suppressor gene PTEN marks the transition from intratubular germ cell neoplasias (ITGCN) to invasive germ cell tumors. Oncogene 2005; 24: 1882-1894.

17. Datta MW, Macri E, Signoretti S, Renshaw AA, Loda M. Transition from in situ to invasive testicular germ cell neoplasia is associated with the loss of p21 and gain of $\mathrm{mdm}-2$ expression. Mod Pathol 2001; 14: 437-442.

18. Voorhoeve PM, le Sage C, Schrier M, Gillis AJ, Stoop H, Nagel R et al. A genetic screen implicates miRNA-372 and miRNA-373 as oncogenes in testicular germ cell tumors. Cell 2006; 124: 1169-1181.

19. Koster R, di Pietro A, Timmer-Bosscha H, Gibcus JH, van den Berg A, Suurmeijer AJ et al. Cytoplasmic p21 expression levels determine cisplatin resistance in human testicular cancer. J Clin Invest 2010; 120: 3594-3605.

20. Vassilev LT, Vu BT, Graves B, Carvajal D, Podlaski F, Filipovic $Z$ et al. In vivo activation of the p53 pathway by small-molecule antagonists of MDM2. Science 2004; 303: 844-848.

21. Kojima K, Konopleva M, Samudio IJ, Shikami M, Cabreira-Hansen M, McQueen T et al. MDM2 antagonists induce p53-dependent apoptosis in AML: implications for leukemia therapy. Blood 2005; 106: 3150-3159.

22. Janz M, Stuhmer T, Vassilev LT, Bargou RC. Pharmacologic activation of p53-dependent and p53-independent apoptotic pathways in Hodgkin/Reed-Sternberg cells. Leukemia 2007; 21: 772-779.

23. Vassilev LT. MDM2 inhibitors for cancer therapy. Trends Mol Med 2007; 13: 23-31.

24. Bauer S, Muhlenberg T, Leahy M, Hoiczyk M, Gauler T, Schuler M et al. Therapeutic potential of Mdm2 inhibition in malignant germ cell tumours. Eur Urol 2010; 57: 679-687.

25. Li B, Cheng $Q$, Li Z, Chen J. p53 inactivation by MDM2 and MDMX negative feedback loops in testicular germ cell tumors. Cell Cycle 2010; 9: 1411-1420.

26. Fulda S, Los M, Friesen C, Debatin KM. Chemosensitivity of solid tumor cells in vitro is related to activation of the CD95 system. Int J Cancer 1998; 76: 105-114.

27. Friesen C, Fulda S, Debatin KM. Cytotoxic drugs and the CD95 pathway. Leukemia 1999; 13: $1854-1858$

28. Timmer T, de Vries EG, de Jong S. Fas receptor-mediated apoptosis: a clinical application? J Pathol 2002; 196: 125-134.

29. Spierings DC, de Vries EG, Vellenga E, de Jong S. The attractive Achilles heel of germ cell tumours: an inherent sensitivity to apoptosis-inducing stimuli. J Pathol 2003; 200: 137-148

30. Spierings DC, de Vries EG, Stel AJ, te Rietstap N, Vellenga E, de Jong S. Low p21Waf1/ Cip1 protein level sensitizes testicular germ cell tumor cells to Fas-mediated apoptosis. Oncogene 2004; 23: 4862-4872. 
31. Lutzker SG, Levine AJ. A functionally inactive p53 protein in teratocarcinoma cells is activated by either DNA damage or cellular differentiation. Nat Med 1996; 2: 804-810.

32. Zamble DB, Jacks T, Lippard SJ. p53-dependent and -independent responses to cisplatin in mouse testicular teratocarcinoma cells. Proc Natl Acad Sci USA 1998; 95 6163-6168.

33. Xia M, Knezevic D, Vassilev LT. p21 does not protect cancer cells from apoptosis induced by nongenotoxic p53 activation. Oncogene 2011; 30: 346-355.

34. Espinosa JM, Verdun RE, Emerson BM. p53 functions through stress- and promoterspecific recruitment of transcription initiation components before and after DNA damage. Mol Cell 2003; 12: 1015-1027.

35. Gomes NP, Espinosa JM. Differential regulation of p53 target genes: it's (core promoter) elementary. Genes Dev 2010; 24: 111-114.

36. Morachis JM, Murawsky CM, Emerson BM. Regulation of the p53 transcriptional response by structurally diverse core promoters. Genes Dev 2010; 24: 135-147.

37. Jiang M, Pabla N, Murphy RF, Yang T, Yin XM, Degenhardt K et al. Nutlin-3 protects kidney cells during cisplatin therapy by suppressing Bax/Bak activation. J Biol Chem 2007; 282 2636-2645.
38. Timmer-Bosscha H, Timmer A, Meijer C, de Vries EG, de Jong B, Oosterhuis JW et al. cisdiamminedichloroplatinum(ii) resistance in vitro and in vivo in human embryonal carcinoma cells. Cancer Res 1993; 53: 5707-5713.

39. Sark MW, Timmer-Bosscha H, Meijer C, Uges DR, Sluiter WJ, Peters WH et al. Cellular basis for differential sensitivity to cisplatin in human germ cell tumour and colon carcinoma cell lines. Br J Cancer 1995; 71: 684-690.

40. Timmer-Bosscha H, de Vries EG, Meijer C, Oosterhuis JW, Mulder NH. Differential effects of all-trans-retinoic acid, docosahexaenoic acid, and hexadecylphosphocholine on cisplatin-induced cytotoxicity and apoptosis in a cisplantin-sensitive and resistant human embryonal carcinoma cell line. Cancer Chemother Pharmacol 1998; 41: 469-476.

\section{(c)}

SOMERIEHTS RES

Cell Death and Disease is an open-access journal published by Nature Publishing Group. This work is licensed under the Creative Commons Attribution-Noncommercial-No Derivative Works 3.0 Unported License. To view a copy of this license, visit http://creativecommons.org/licenses/by-nc-nd/3.0/

Supplementary Information accompanies the paper on Cell Death and Disease website (http://www.nature.com/cddis) 